\title{
artigo
}

Santos, D.M.V.; Guimaraes da Silva, L.; Pardi, P.C.; Santos, G.A.A.; Segura, M.E.A.; Xavier, J.S.;

Carcinoma mamário: Classificação molecular utilizando a imuno-histoquímica

\section{Carcinoma mamário: Classificação molecular utilizando a imuno-histoquímica}

\author{
Breast carcinoma: Molecular classification using immunohistochemistry \\ Carcinoma de mama: clasificación molecular mediante inmunohistoquímica
}

\begin{abstract}
RESUMO
Objetivo: Classificar diferentes tipos histológicos para carcinoma mamário, do tipo invasivo, com auxílio da imuno-histoquímica. Métodos: Selecionamos 3033 casos positivos, destes, 2433 do tipo invasivo que foram classificados em quatro grupos: Luminal A, luminal B, HER2-eriched e Basal like (triplo negativo). Realizamos um estudo retrospectivo observacional entre os anos de 2011 e 2016Resultados: 2062 (84,4\%) dos laudos revisados, foram referentes a CDI SOE, seguido pelos tipos em ordem decrescente: CLI $(11,3)$; carcinoma micropapilar invasivo $(1,11 \%)$ e os demais tipos com incidência menor que $1 \%$. Conclusão: Este estudo permitiu a diferenciação histológica e caracterização do perfil IHQ do carcinoma mamário invasivo ocorridos em um período de 6 anos no Distrito Federal.
\end{abstract}

DESCRITORES: Mama; Carcinoma, Câncer, Neoplasia, Imuno-histoquímica.

\section{ABSTRACT}

Objective: To classify different histological types for mammary carcinoma, invasive type, with the aid of immunohistochemistry. Methods: We selected 3033 positive cases, 2433 of the invasive type, which were classified into four groups: Luminal $A$, luminal B, HER2-eriched, and Basal like (triple negative). We conducted a retrospective observational study between the years 2011 and 2016. Results: 2062 (84.4\%) of the related to CDI SOE, following the items in decreasing order: CLI (11.3); Invasive micropapillary carcinoma $(1.11 \%)$ and other types with incidence less than $1 \%$. Conclusion: This study allowed a histological differentiation and characterization of the IHQ profile of invasive mammary carcinoma over a 6 years in the Federal District.

DESCRIPTORS: Breast, Carcinoma, Cancer, Neoplasm, Immunohistochemistry.

\section{RESUMEN}

Objetivo: Clasificar diferentes tipos histológicos del carcinoma de mama de tipo invasivo, con ayuda de inmunohistoquímica. Métodos: Se seleccionaron 3033 casos positivos, de estos 2433 del tipo invasivo, los cuales fueron clasificados en cuatro grupos: Luminal A, Luminal B, HER2-eriched y Basal like (triple negativo). Realizamos un estudio observacional retrospectivo entre los años 2011 y 2016 Resultados: 2062 (84,4\%) de los informes revisados estaban relacionados con CDI SOE, seguidos de los tipos en orden decreciente: CLI (11,3); carcinoma micropapilar invasivo $(1,11 \%)$ y los otros tipos con una incidencia inferior al $1 \%$. Conclusión: Este estudio permitió la diferenciación histológica y caracterización del perfil IHC del carcinoma invasivo de mama que se presentó en un período de 6 años en el Distrito Federal.

DESCRIPTORES: mama; Carcinoma, cáncer, neoplasia, inmunohistoquímica

RECEBIDO EM: 11/08/2020 APROVADO EM: 28/09/2020

\section{David Marlon Vieira Santos}

Biólogo. Especialista em Anatomia Humana pela Pontifícia Universidade Católica de Goiás (GO), com foco em docência (2018). ORCID: 0000-0002-2405-9475

\section{Luana Guimaraes da Silva}

Enfermeira. Graduação em Enfermagem pela FACESA.

ORCID: 0000-0001-6009-1037

\section{Paulo Celso Pardi}

Biomédico (UMC), Especialista em Gerontologia (UNIDERP) e Mestre e Doutor em Morfologia (UNIFESP).

ORCID: 0000-0001-5903-6452 


\section{Gustavo Alves Andrade dos Santos}

Farmacêutico Bioquímico; Pós graduado em Farmácia Hospitalar; Doutor em Biotecnologia; Mestre em Farmácia. Pós doutorando em Anatomia e Cirurgia pela Faculdade de Medicina da USP de Ribeirão Preto.

ORCID: 0000-0002-3326-5537

\section{Marcos Emanuel de Alcântara Segura}

Médico. Graduação em Medicina pela Universidade de Brasília (2003), mestrado em Ciências Médicas pela Universidade de Brasilia (2008).

ORCID: 0000-0001-6607-211X

\section{Joaquim da Silva Xavier}

Biólogo. Graduação em Biologia pela Universidade Católica de Brasília (2002) e mestrado em Patologia Molecular pela Universidade de Brasília (2006).

ORCID: 0000-0001-6623-8597

\section{INTRODUÇÃO}

câncer de mama representa um dos principais problemas de saúde pública no mundo. Excetuando-se a pele, é a neoplasia mais comum entre as mulheres. Em 2008, cerca de 13,7\% dos óbitos por câncer no mundo foram em decorrência de carcinoma mamário. (Gebrim $\left.^{7}, 2015\right)$. No Brasil, essa neoplasia é a principal causa de óbito entre mulheres. Em 2010, o Ministério da Saúde estimou aproximadamente 49.240 novos casos no país, sendo mais comum em pacientes pós menopausa, com faixa etária entre 50 e 69 anos. (Cintra $\left.{ }^{3}, 2012\right)$. Trata-se de uma doença heterogênea com diferentes apresentações histológicas, moleculares e clínicas (Vinay K, et al. ${ }^{18}$ 2010).

$\mathrm{O}$ diagnóstico e caracterização do câncer de mama se inicia com a avaliação morfológica por histopatologia convencional. Resumidamente, após retirada do tecido suspeito o mesmo é processado e cortado em finas fatias de 3 a 5 micras, montados em lâminas que são coradas com hematoxilina e eosina. Essas lâminas são examinadas pelo médico patologista que define se há malignidade na amostra e, caso positivo, faz a classificação histológica da neoplasia. A Organização Mundial de Saúde (O.M.S.) prevê vários tipos histológicos de câncer de mama (Lakhani SR, et al. ${ }^{10}$ 2012.), sendo o mais comum o chamado carcinoma mamário invasivo, sem tipo especial (carcinoma ductal infiltrante, sem outras especificações / CDI-SOE) com cerca de 5080\% dos casos. Em seguida temos o carcinoma lobular infiltrante (CLI) que representa de 5 a $15 \%$ dos casos (Tabela 1).

Depois de concluído o exame histopatológico convencional, faz-se necessário a pesquisa da expressão do Receptor de Estrógeno (RE), Receptor de Progesterona (RP) e da proteína do gene HER2/neu (HER2). O método mais difundido para esse fim é a imuno-histoquímica (IHQ), que permite determinar in situ, ou seja, no próprio tecido, a expressão proteica no contexto da morfologia tumoral. $\mathrm{O}$ uso rotineiro da IHQ para análise da expressão do RE, RP e HER2 fornece informações críticas sobre fatores prognósticos $\mathrm{e}$ preditivos para os carcinomas mamários e guiará a terapia subsequente (Rakha EA, et al. $\left.{ }^{14} 2010\right)$.

\section{TABELA 1 Prevalência de tipos histológicos de carcinomas mamários} reconhecidos pela O.M.S.

\begin{tabular}{|c|c|c|c|c|}
\hline Tipo Histológico & $\begin{array}{l}\text { Prevalência (\%) } \\
\text { ROSEN (2001) }\end{array}$ & $\begin{array}{l}\text { Prevalência (\%) } \\
\text { ELLIS (2003) }\end{array}$ & $\begin{array}{c}\text { Prevalência (\%) } \\
\text { RAKHA } \\
(2006,2008)\end{array}$ & $\begin{array}{c}\text { Prevalência (\%) } \\
\text { LOUWMAN } \\
\text { (2007) }\end{array}$ \\
\hline CDI-SOE & $65-80$ & $50-80$ & 56.4 & 78 \\
\hline CLI & 5 & $5-15$ & 15 & 11.1 \\
\hline Ca. tubular & $<2$ & $<2$ & 4.4 & 2.2 \\
\hline Ca.cribriforme & $<4$ & $0,8-3,5$ & 0,6 & 0,3 \\
\hline $\begin{array}{l}\text { Ca. micropapilar } \\
\text { invasivo }\end{array}$ & $<2,7$ & $<2$ & - & - \\
\hline Ca. mucinoso & $<2$ & 2 & 1.4 & 2.2 \\
\hline Ca. metaplásico & $<5$ & $<1$ & - & 0,2 \\
\hline $\begin{array}{l}\text { Ca. com caracte- } \\
\text { rísticas medulares }\end{array}$ & $<5-7$ & $1-7$ & 2,6 & 1,1 \\
\hline Ca. apócrino & $<1-4$ & $<4$ & - & - \\
\hline $\begin{array}{l}\text { Ca. com células e } \\
\text { anel de sinete }\end{array}$ & - & - & - & - \\
\hline $\begin{array}{l}\text { Adenomioepitelio- } \\
\text { ma com carcinoma }\end{array}$ & - & - & - & - \\
\hline $\begin{array}{l}\text { Ca. adenoide } \\
\text { cístico }\end{array}$ & - & 0,1 & - & 0,1 \\
\hline $\begin{array}{l}\text { Ca. com diferen- } \\
\text { ciação neuroen- } \\
\text { dócina }\end{array}$ & - & - & - & - \\
\hline
\end{tabular}




\section{artigo}

Santos, D.M.V.; Guimaraes da Silva, L.; Pardi, P.C.; Santos, G.A.A.; Segura, M.E.A.; Xavier, J.S.;

Carcinoma mamário: Classificação molecular utilizando a imuno-histoquímica

\begin{tabular}{|c|c|c|c|c|}
\hline Ca. secretório & - & $<0,15$ & - & - \\
\hline Ca. papilar invasivo & - & - & - & - \\
\hline $\begin{array}{l}\text { Ca. de células } \\
\text { acinares }\end{array}$ & - & - & - & - \\
\hline $\begin{array}{l}\text { Ca. muco-epider- } \\
\text { móide }\end{array}$ & - & - & - & - \\
\hline Ca. polimórfico & - & - & - & - \\
\hline Ca. oncocítico & - & - & - & - \\
\hline Ca. rico em lipídeos & $<1$ & $<1-6$ & - & - \\
\hline $\begin{array}{l}\text { Ca. rico em glico- } \\
\text { gênio }\end{array}$ & $<1-3$ & $1-3$ & - & - \\
\hline Ca. sebáceo & - & - & - & - \\
\hline
\end{tabular}

A partir dos anos 2000, os trabalhos liderados por Perou e Sorlie (Perou CM, et al. ${ }^{11}$ 2000); (Sorlie T, et al. ${ }^{15}$ 2001); (Sorlie T, et al. ${ }^{16} 2003$ ) utilizando perfil de expressão genica (PEG) identificou 5 subtipos moleculares de carcinoma mamário: luminal A, luminal B, normal breast-like, HER2-enriched, e basal-like. Posteriormente, foi verificado que cada classe molecular se correlaciona a diferentes sobrevidas e respostas à quimioterapia (QT). Várias outras classificações foram propostas, porém foram constantes os grupos Luminal A, Luminal B, HER2-enriched e Basal-like.

Em resumo, o subtipo molecular Luminal A (LumA), é caracterizado pela similaridade que células neoplásicas apresentam com células normais encontradas nos ductos mamários. Esse subtipo representa aproximadamente $60 \%$ dos tumores mamários, e possui melhor prognóstico. Em sua maioria, são menores que os demais, e caracterizados pela elevada expressão de $\mathrm{RE}$, com baixo índice proliferativo, e baixo grau histológico. É o grupo com melhor benefício para terapia hormonal, e benefício limitado para QT (Cirqueira $\left.{ }^{4}, 2011\right)$.

Os tumores do Luminal B (LumB), à semelhança dos Luminais $A$ possuem expressão de RE. E se diferem daqueles pelo alto índice proliferativo, e associação com a expressão de HER2. Apresentam um maior índice de proliferação, e seu prognóstico é pior se comparado com os demais. Os tumores LumB apresentam uma maior taxa de recorrência e menor sobrevida. É esperada uma melhor resposta à QT e pior resposta à hormonioterapia (Cirqueira ${ }^{4}, 2011$ ).

Como dito acima, os tumores Luminal A e Luminal B são caracterizados pela expressão de receptores hormonais, principalmente RE. Os outros tipos tumorais, caracterizados pela ausência de expressão desses receptores são os HER2-enriched e Basal-Like. O HER2-enriched é caracterizado pela elevada expressão do gene HER2/neu e de outros genes relacionados. Geralmente esses tumores são poucos diferenciados e possuem uma alta taxa proliferativa, sendo associados a grande frequência de metástase cerebral. Pacientes com essa classificação possuem resistência intrínseca às terapias endócrinas, mas se beneficiam com a terapia antiHER2 e QT tradicional (Cirqueira ${ }^{4}, 2011$ ).

Por último, os tumores Basal-Like compreendem cerca de $13 \%$ a $25 \%$ dos tumores mamários. Esse subgrupo apresenta negatividade para receptores hormonais e HER2, alto grau histológico, alta taxa de proliferação e estão associados a menor sobrevida do paciente. Representam um grupo de tumores sem tratamento específico, porém quimiossensível (Vinay $\mathrm{K}^{18}$, et al. 2010).

A contribuição de métodos que utiliza perfil de expressão gênica teve inigualável importância para o entendimento do carcinoma mamário, porém o emprego desses métodos não é prático do ponto de vista econômico nem técnico, necessitando de laboratórios especializados de alto custo. Em razão disso, vários investigadores propuseram que o painel de IHQ pode substituir os métodos moleculares (Tang P; Tse GM17. 2015). Em 2013, a convenção de St. Gallen recomendou uma classificação molecular do câncer de mama baseada em IHQ (Goldhirsh A, et al8. 2013). Essa classificação utiliza uma combinação dos resultados para RE, RP, HER2 e Ki67 (Tabela 2).

O presente trabalho tem o objetivo de correlacionar os subtipos histológicos de carcinomas mamários com os tipos moleculares definidos pela IHQ.

\section{MÉTODOS}

Foi realizado um estudo retrospectivo e observacional com revisão de 3.033 laudos de IHQ de mama de um laboratório de anatomia patológica de Brasília-DF (Laboratório Lâmina) nos anos de 2011 a 2015. Foram excluídos da análise casos de neoplasia "in situ" a fim de preservar apenas os casos de neoplasia invasiva, totalizando 2433 casos analisados.

Os casos foram morfologicamente avaliados por histomorfologia convencional e a reação de imuno-histoquímica foi revelada com o sistema livre de biotina NOVOLINK (LeicaBiosistems). Na pesquisa dos receptores hormonais utilizou-se os seguintes anticorpos monoclonais: RE clone 6F11 (LeicaBiosistems) na diluição de 1:400; RP o clone 16 (LeicaBiosistems) na diluição de 1:800. A gradação dos receptores hormonais foi realizada usando-se o método de Allred (Allred DC, et al. 1998) O score de Allred avalia a proporção de células positivas juntamente com a intensidade da reação. O score varia de 0 a 8 , sendo que os casos maiores que 3 são considerados positivos.

$\mathrm{O}$ anticorpo usado para evidenciar a expressão de HER2 foi o SP3 (Epitomics) na diluição de 1:500. A avaliação da expressão do HER-2 seguiu as orientações da associação americana de oncologia clínica e colégio americano de patologistas (ASCO/ CAP). (Wolff AC, et a ${ }^{20} .2013$ ) Os casos identificados na imunohistoquímica como duvidosos foram encaminhados para pes- 
quisa de amplificação genica por hibridização "in situ" por fluorescência (FISH).

$\mathrm{O}$ anticorpo utilizado para o Ki67 foi o SP6 (Cell Marque) na diluição de 1:500. A leitura da reação foi realizada manualmente com registro da proporção de células positivas para a marcação.

Baseado nas informações contidas nos laudos, cada caso recebeu uma classificação molecular baseada na proposta de StGallen 2015 (TABELA 2).

Os subtipos histológicos foram então correlacionados com o subtipo molecular.

O software Microsoft Excel 2013 foi utilizado para processamento dos dados.

\section{RESULTADOS}

Do total de 3.033 laudos revisados, 2433 eram referentes a carcinoma invasivo e continham todas as informações para serem incluídos no estudo. O tipo histológico mais comum foi o Carcinoma ductal infiltrante, S.O.E (CDI-SOE) (Carcinoma mamário invasivo sem tipo especial na classificação OMS 2012) totalizando 2062 casos $(84,4 \%)$. Os demais tipos histológicos em ordem decrescente foram: carcinoma lobular invasivo $(11,3 \%)$, carcinoma micropapilar invasivo $(1,11 \%) \mathrm{e}$ com prevalência menor que $1 \%$ observa-

TABELA 2.Classificação molecular do câncer de mama baseado em IHQ. Proposta do St Gallen International Expert Consensus on the Primary Therapy of Early Breast Cancer (Coates AS, et al. 2015).

\section{TIPO MOLECULAR}

Luminal A (LumA)

Luminal B HER2- (LumB HER2-)

Luminal B HER2+ (LumB HER2+)

HER2-neu non-luminal (HER2 N/L)

Triple Negative Breast Cancer (TNBC)

\section{CRITERIO}

$$
\begin{gathered}
\mathrm{RE}+\text { e /ou RP+ HER2 - Ki67\% baixo* } \\
\mathrm{RE}+\text { e /ou RP+ HER2 - Ki67\% alto* } \\
\text { RE+ e /ou RP+ HER2 + } \\
\text { RE - / RP- HER2 + }
\end{gathered}
$$$$
\text { RE- / RP- HER2 - }
$$

* Para a definição do ponto de corte para o Ki67, foi calculado a mediana da expressão nos casos positivos para RE (20\%). Valores $\leq 20 \%$ foram considerados baixo, valores $>20 \%$ foram considerados alto.

\section{TABELA 3}

Tipo Histológico

CDI-SOE

grau 1

grau 2

grau 3

CLI-SOE

Ca. micropapilar invasivo

Ca. mucinoso

Ca. invasivo ductal e lobular

Ca. tubular

Ca. papilar invasivo

Ca. Apócrino

Ca. Metaplásico

Ca. Túbulo-lobular

Ca. Adenóide-Cístico

Ca. com caract. medulares

\section{LumA}

$975(47,3 \%)$

$280(92,7 \%)$

$647(54,9 \%)$

$48(6,9 \%)$

$213(76,6 \%)$

$5(18,5 \%)$

20(83\%)

$3(33.3 \%)$

$9(100,0 \%)$

$4(66,7 \%)$

$1(16,7 \%)$

0

$3(100,0 \%)$

0

0

\begin{abstract}
LumB/
\end{abstract}
HER2-

$492(23,9 \%)$

$11(3,6 \%)$

$297(25,2 \%)$

$266(38,1 \%)$

$53(19,1 \%)$

$16(59,3 \%)$

$3(12,5 \%)$

3(33.3\%)

0

$2(33,3 \%)$

1(16,7\%)

$1(14,3 \%)$

0

0

$1(50,0 \%)$
LumB/ HER2+

$186(9 \%)$

$7(2,3 \%)$

$110(9,3 \%)$

$81(11,6 \%)$

$7(2,5 \%)$

$3(11,1 \%)$

$1(4,2 \%)$

$1(11,1 \%)$

0

0

0

0

0

0

0
HER2 N/L

$143(6,9 \%)$

$1(0,3 \%)$

$62(5,3 \%)$

$83(11,9 \%)$

0

$1(3,7 \%)$

0

$1(11,1 \%)$

0

0

$1(16,7 \%)$

0

0

0

0 mos carcinoma mucinoso, carcinoma tubular, carcinoma invasivo ductal e lobular, carcinoma papilar invasivo, carcinoma apócrino, carcinoma metaplásico, carcinoma túbulo-lobular, carcinoma adenoide-cístico, carcinoma com características medulares, carcinoma ductal com diferenciação neuro-endócrina, carcinoma secretor e carcinoma cribriforme.

A tabela 3 relaciona os tipos histológicos com a respectiva classificação molecular por IHQ. Para os casos CDI-SOE, observa-se que graus histológicos menores estavam relacionados a maior proporção de casos LumA. Por exemplo, os casos CDI-SOE grau1, apresentaram proporção de casos LumA de 92,7\%, os casos CDI-SOE grau 2 a proporção de casos LumA foi de $54,9 \%$ e para o grau 3 apenas $6,9 \%$. O carcinoma micropapilar invasivo foi majoritariamente classificado como LumB. O carcinoma mucinoso teve $83 \%$ dos casos classificados como LumA. Carcinoma túbulo-lobular e cribriforme tiveram todos os casos classificados como LumA. Carcinoma adenoide-cístico teve todos os casos classificados como TNBC. A maioria dos casos de carcinoma metaplásico foi classificado como TNBC. 


\section{artigo}

Santos, D.M.V.; Guimaraes da Silva, L.; Pardi, P.C.; Santos, G.A.A.; Segura, M.E.A.; Xavier, J.S.;

Carcinoma mamário: Classificação molecular utilizando a imuno-histoquímica

$\begin{array}{lcccccc}\text { Ca. com dif neuroendócrina } & 2(66,6 \%) & 1(33,3 \%) & 0 & 0 & 0 & 3 \\ \text { Ca. Secretor } & 0 & 1(100,0 \%) & 0 & 0 & 0 & 1 \\ \text { Ca. Cribriforme } & 2(100,0 \%) & 0 & 0 & 0 & 0 & 2 \%\end{array}$

Figura 1 Distribuição dos tipos histológicos de acordo com a classificação molecular. A espessura da linha indica a proporção de casos relacionados a determinada classificação

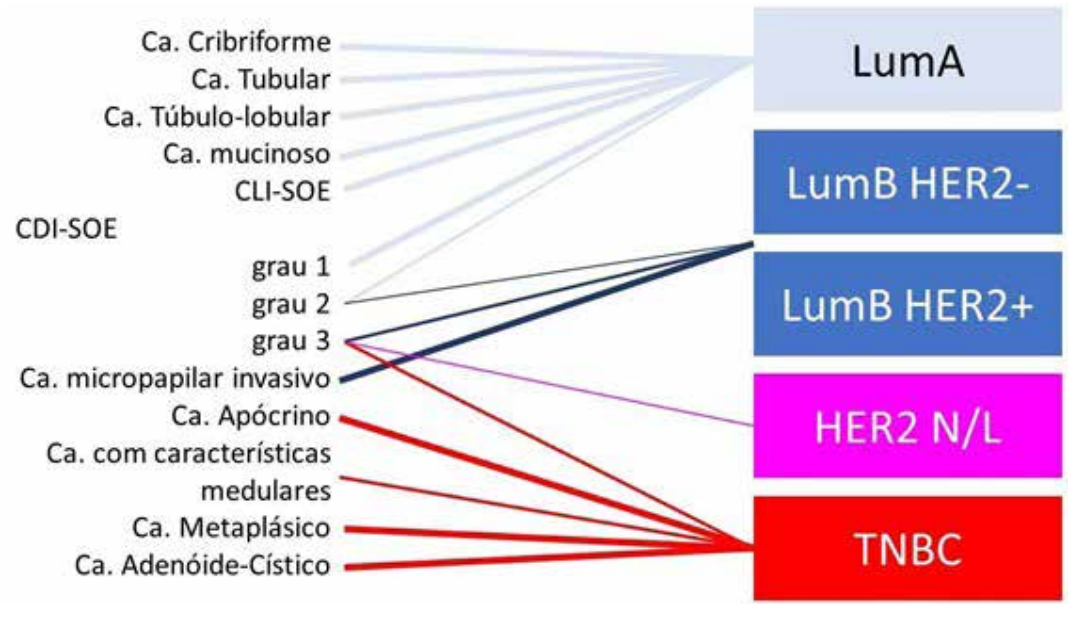

\section{DISCUSSÃO}

Correlacionamos neste estudo vários tipos histológicos de carcinoma mamário com sua classificação molecular, por meio de imuno-histoquímica. A avalição do tipo histológico associada à pesquisa de expressão de receptores hormonais, HER2 e Ki67 são fatores prognósticos e preditivos levados em consideração no gerenciamento de terapias mais adequadas para cada tipo tumoral.

O tipo histológico mais comum observado neste estudo foi o carcinoma ductal infiltrante, sem outras especificações (CDI-SOE) (Carcinoma mamário invasivo sem tipo especial na classificação OMS 2012). Constatamos o fato de que esses tumores não são um grupo homogêneo, mas que podem ser melhores classificados de acordo com o grau histológico. Os CDI-SOE grau 1 foram majoritariamente classificados como LumA. O CDI-SOE grau 2, se comportou como o tipo histológico mais heterogêneo da amostra. Metade dos casos $(54,9 \%)$ foram classificados como
LumA, sendo os casos restantes distribuídos pelos grupos LumB(34,5\%) HER2 $\mathrm{N} / \mathrm{L}(5 \%)$ e TNBC(5\%). Carcinomas do tipo CDI-SOE grau 3 tiveram baixa prevalência de casos

LumA sendo a maioria dos casos distribuídos entre LumB, HER2 N/L e TNBC. Esse fato demonstra que o grau histológico é capaz de sintetizar fatores da biologia tumoral, acarretando em diferentes classificações moleculares.

O segundo tipo histológico mais comum, carcinoma lobular infiltrante, foi classificado predominantemente como LumA. Alguns tipos histológicos tiveram todos os casos classificados no mesmo grupo molecular. O carcinoma adenoide-cístico teve todos seus casos classificados como TNBC. Já os carcinomas cribriforme, tubular e túbulo-lobular foram todos classificados como LumA. Os casos de carcinoma micropapilar invasivo foram predominantemente do tipo LumB. Os carcinomas mucinosos foram predominantemente LumA (83\%) mas um pequeno grupo foi classificado como LumB HER2- (12,5\%).
Em uma abordagem semelhante, que usou IHQ para classificação molecular dos subtipos histológicos, Alvarenga e cols. (Alvarenga CA, et al. 2012) chegaram a resultados semelhantes aos apresentados aqui. Estudando um grupo previamente selecionado de subtipos especiais, foi verificado que o subtipo tubular pertencia à classificação LumA. O subtipo mucinoso se distribuiu em LumA e LumB. Os subtipos metaplásicos e medulares foram predominantemente do padrão basal-like (TNBC na classificação do presente estudo). Por fim, o subtipo micropapilar apresentou um padrão LumB predominante.

Weigelt e cols ${ }^{19}$ (2008), em um estudo envolvendo a análise imunohistoquímica e transcriptômica de vários subtipos moleculares, verificaram que alguns subtipos histológicos constituem entidades específicas com padrões próprios de expressão molecular e atividade transcricional. Foi verificado que em nível transcriptômico, os subtipos histológicos são mais homogêneos entre si do que os CDI-SOE e CLI-SOE. Os subtipos histológicos tubular e mucinoso demonstraram um padrão luminal claro enquanto o adenoide-cístico, medular e metaplásico expressaram um fenótipo basallike. No presente estudo foi possível reproduzir esse achado, caracterizando o subtipo CDI-SOE, principalmente o grau 2, como uma classe de tumor heterogênea, com ampla distribuição pelos tipos moleculares.

A Figura 1 apresentam resumo das correlações entre os subtipos histológicos e moleculares observados neste estudo, que reproduz os achados da literatura. Temos que os tumores de baixo grau (Carcinoma cribriforme, tubukar, túbulo-lobular, mucinoso, CLI-SOE e os CDI-SOE grau 1) são classificados predominantemente como LumA. O carcinoma micropapilar invasivo é fortemente relacionado ao tipo molecular LumB. Vários tipos especiais se classificam como TNBC (Carcinoma apócrino, com características medulares, 
metaplásico e adenoide-cístico). Os CDISOE graus 2 e 3 são muito heterogêneos, mas a proporção de casos LumA decresce à medida que se aumenta o grau histológico.

\section{CONCLUSÃO}

De uma maneira geral, este trabalho demonstrou a grande prevalência, em pacientes do sexo feminino, do carcinoma mamário invasivo sem outras especificações (CDI-SOE), seguido pelo carcinoma lobular infil- trante sem outras especificações (CLI-SOE) assim como, os demais tipos histológicos que também apresentaram resultados semelhantes aos já descritos na literatura.

Neste contexto a utilização rotineira da imuno-histoquímica permitiu de forma prática e econômica, a partir da expressão do RE, RP e HER2, a classificação molecular conforme proposta por St. Gallen em 2013. Também foi possível demonstrar a heterogeneidade da doença, e que o conhecimento acerca de fatores prognós- ticos e preditivos, implica diretamente no gerenciamento de terapias mais adequadas, com menores efeitos adversos.

Através desse trabalho podemos perceber a importância da IHQ em diagnosticar o subtipo molecular, proporcionando o conhecimento necessários para tratamentos individualizados, baseados em novos conhecimentos moleculares, entretanto, novos estudos são necessários afim de determinar futuros alvos terapêuticos nos diferentes tipos histológicos do carcinoma mamário.

\section{REFERÊNCIAS}

1. ALLRED DC, HARVEY JM, et al. Prognostic and predictive factors in breast cancer by immunohistochemical analysis. Mod Pathol. 1998; 11: 155-168

2. ALVARENGA CA, PARAVIDINO PI, ALVARENGA M, et al. Reappraisal of immunohistochemical profiling of special histological types of breast carcinomas: a study of 121 cases of eight different subtypes. Journal of clinical pathology. 2012 Dec;65(12):1066-71. PubMed PMID: 22944625. Epub 2012/09/05. eng.

3. CINTRA JRD, TEIXEIRA MTB, DINIZ RW, et al. Perfil imunohistoquímico e variáveis clinicopatológicas no câncer de mama.Rev. Assoc. Med. Bras 2012; 58(2): 178-187

4. CIRQUEIRA MB, MOREIRA MAR, SOARES LR, JÚNIOR RF. Subtiposmoleculares do câncer de mama. Femina, 2011; vol. 39; $n^{\circ} 10 ; 499-503$.

5. COATES AS, WINER EP, GOLDHIRSCH A, GELBER RD, GNANT M, PICCART-GEBHART $M$, et al. Tailoring therapies-improving the management of early breast cancer: St Gallen International Expert Consensus on the Primary Therapy of Early Breast Cancer 2015. Ann Oncol. 2015; 26: $1533 e 46$.

6. Ellis P., Schnitt S.J., Sastre-Garau X. Invasive breast carcinoma. In: Tavassoli, F.A., Devilee, P. (Eds.), WHO Classification of Tumours Pathology and Genetics of Tumours of the Breast and Female Genital Organs. 2003. Lyon Press, Lyon.

7. GEBRIM LH, SHIDA JY, HEGG R, TOPIS T, MATTAR A. Avaliação do tempo de inicio do tratamento, estadiamentohistopatológico e positividade dos biomarcadores (RE, RP, HER-2) em 3.566 pacientestratadaspelo SUS no período de 2012 a 2014, no Hospital PérolaByington. Rev. Bras. Mastologia. 2014, 24(3): 65-69.

8. GOLDHIRSH A, WINER EP, COATES AS, et al. Personalizing the treatment of women with early breast cancer: highlights of the St. Gallen International Expert Consensus on the primary therapy of early breast cancer 2013. Ann Oncol. 2013; 24(9):2206- 2223.

9. HAMMOND ME, HICKS DG, DOWSETT M, et al. Recommendations for human epidermal growth factor receptor 2 testing in breast cancer: American Society of Clinical Oncology/College of American Pathologists clinical practice guideline update. Journal of clinical oncology: official journal of the American Society of
Clinical Oncology. 2013 Nov 1;31(31):3997-4013. PubMed PMID: 24101045. Epub 2013/10/09. eng.

10. LAKHANI SR, ELLIS IO, SCHNITT SJ, et al. World Health Organization Classification of Tumours of the Breast. Lyon, France: IARC Press; 2012.

11. PEROU CM, SORLIE T, EISEN MB, et al. Molecular portrait of human breast tumours. Nature. 2000; 406 (6797):747-752.

12. Rakha E.A., El-Sayed M.E., Menon S. Histologic grading is an independent prognostic factor in invasive lobular carcinoma of the breast. Breast Cancer. 2008. Res. Treat. 111, 121e127.

13. Rakha E.A., Putti T.C., Abd El-Rehim D.M. Morphological and immunophenotypic analysis of breast carcinomas with basal and myoepithelial differentiation. 2006. J. Pathol. 208, 495e506.

14. RAKHA EA, REIS-FILHO JS, ELLIS IO. Combinatorial biomarker expression in breast cancer. Breast Cancer Res Treat. 2010;120(2):293-308.

15. SORLIE T, PEROU CM, TIBSHIRANI R, et al. Gene expression patterns of breast carcinomas distinguish tumor subclasses with clinical implications. Proc Natl AcadSci U S A. 2001; 98 (19):10869-10874.

16. SORLIE T, TIBSHIRANI R, PARKER J, et al. Repeated observation of breast tumor subtypes in independent gene expression data sets. Proc Natl AcadSci U S A 2003;100(14):8418-8423

17. TANG P, TSE GM. Immunohistochemical Surrogates for Molecular Classification of Breast Carcinoma: A 2015 Update. Arch Pathol Lab Med. 2016 Aug; 140(8):806-14. doi: 10.5858/arpa.2015-0133-RA.

18. VINAY et al. Robbins e CotranPatologia: bases patológicas das doenças. 8 ed. Rio de Janeiro: Elsevier, 2010. 1458 p.

19. WEIGELT B, HORLINGS HM, KREIKE B, et al. Refinement of breast cancer classification by molecular characterization of histological special types. J Pathol. 2008 Oct;216(2):141-50. PubMed PMID: 18720457. Epub 2008/08/23.

20. WOLFF C.A; HAMMOND E.H; KIMBERLY $H$, et al. HumanEpidermalGrowthFactor Receptor 2 Testing in BreastCancer: American SocietyofClinicalOncology/CollegeofAmericanPathologistsClinicalPracticeGuidelineFocused Update. JournalofClinicaIOncology.Vol 36. N² 20. JUL 10, 2018. 